Z. Epileptol. 2022 · 35:169-177 https://doi.org/10.1007/s10309-021-00434-z Online publiziert: 13. Juli 2021

(๑) Der/die Autor(en) 2021

\section{Klinische Charakteristika und Lebensqualität beim Dravet- Syndrom: Ergebnisse der deutschen Kohorte des „Dravet syndrome caregiver survey" (DISCUSS)}

\author{
A. Strzelczyk ${ }^{1,2,3} \cdot$ L. Lagae $^{4} \cdot$ G. Kurlemann ${ }^{5} \cdot$ S. Flege ${ }^{6} \cdot$ T. Bast $^{7,8} \cdot$ T. Polster $^{9}$. \\ M. Pringsheim ${ }^{10,11} \cdot$ S. von Spiczak ${ }^{12} \cdot$ P. Hipp ${ }^{13} \cdot$ S. Schubert-Bast ${ }^{2,14,15}$ \\ 'Epilepsiezentrum Frankfurt Rhein-Main, Zentrum der Neurologie und Neurochirurgie, \\ Universitätsklinikum Frankfurt, Goethe-Universität Frankfurt, Frankfurt am Main, Deutschland; ${ }^{2}$ LOEWE \\ Center for Personalized Translational Epilepsy Research (CePTER), Goethe-Universität Frankfurt, Frankfurt \\ am Main, Deutschland; ${ }^{3}$ Epilepsiezentrum Hessen und Klinik für Neurologie, Philipps-Universität \\ Marburg, Marburg (Lahn), Deutschland; ${ }^{4}$ Department of Development and Regeneration, University \\ Hospitals KU Leuven, Leuven, Belgien; ${ }^{5}$ Klinik für Kinder- und Jugendmedizin, Bonifatius-Hospital Lingen, \\ Lingen (Ems), Deutschland; ${ }^{6}$ Dravet-Syndrom e. V., Markkleeberg, Deutschland; ' ${ }^{7}$ Epilepsiezentrum Kork, \\ Kehl-Kork, Deutschland; ${ }^{8}$ Medizinische Fakultät, Universität Freiburg, Freiburg i. Br., Deutschland; \\ ${ }^{9}$ Kinderepileptologie, Epilepsiezentrum Bethel, Krankenhaus Mara, Bielefeld, Deutschland; ${ }^{10} \mathrm{Klinik}$ für \\ angeborene Herzfehler und Kinderkardiologie, Deutsches Herzzentrum München, München, \\ Deutschland; "Klinik für Neuropädiatrie und Neurologische Rehabilitation, Epilepsiezentrum für Kinder \\ und Jugendliche, Schön Klinik Vogtareuth, Vogtareuth, Deutschland; ${ }^{12}$ Norddeutsches Epilepsiezentrum \\ für Kinder und Jugendliche, Kiel-Raisdorf, Deutschland; ${ }^{13}$ saproma Healthcare Consulting \& Management \\ GbR, Roetgen, Deutschland; ${ }^{14}$ Klinik für Neuropädiatrie, Goethe-Universität Frankfurt, Frankfurt am Main, \\ Deutschland; ${ }^{15}$ Epilepsiezentrum Frankfurt Rhein-Main, Zentrum für Neurologie und Neurochirurgie, \\ Goethe-Universität Frankfurt, Frankfurt am Main, Deutschland
}

Erstveröffentlichung in Monatsschrift Kinderheilkunde. https://doi.org/10.1007/s00112021-01153-5

\section{Hintergrund und Fragestellung}

Das Dravet-Syndrom (DS), erstmals beschrieben von und benannt nach Charlotte Dravet $\left({ }^{*} 1936\right)$ und früher als "schwere infantile myoklonische Epilepsie" (SMEI) bekannt, ist ein seltenes, in der frühen Kindheit beginnendes therapierefraktäres Epilepsiesyndrom, das häufig mit bleibenden schwerwiegenden Einschränkungen in der kognitiven, motorischen, psychomotorischen und neurologischen Entwicklung einhergeht [1]. Das DS wird primär klinisch diagnostiziert, unterstützt von einer Gendiagnostik zur Identifikation einer Mutation im SCN1A-Gen, die bei über $80 \%$ der Betroffenen vorliegt [2]. Die Inzidenz wird in Europa auf etwa 1 zu 15.000 Lebendgeburten und die Prävalenz auf etwa 2 zu 100.000 geschätzt [3-6].

Das DS manifestiert sich im ersten Lebensjahr bei zunächst gesunden Kindern zumeist in Form fieberinduzierter, generalisierter oder halbseitiger tonisch-klonischer oder klonischer Anfälle mit einem hohen Status-epilepticus-Risiko. Im weiteren Krankheitsverlauf treten dann zusätzliche Anfallstypen wie Myoklonien, atypische Absencen oder fokale Anfälle auf. Nach dem fünften Lebensjahr können die Anfallsfrequenz und Anfallsschwere abnehmen. Das DS ist mit einem hohen Risiko eines vorzeitigen Todes durch SUDEP (plötzlicher unerwarteter Tod bei Epilepsie), Status epilepticus oder Unfälle verbunden $[1,2]$.

Während das Ziel der antiepileptischen Therapie üblicherweise eine komplette Anfallsfreiheit ist [2], lässt sich diese bei DS nur in den wenigsten Fällen erreichen. Therapeutisch stehen v.a. die Antiepileptika (,antiepileptic drugs" [AED]) Valproat, Kaliumbromid, Clobazam und Topiramat, das speziell für das DS zugelassene Stiripentol 
in Kombination mit Clobazam und Valproat, seit 2019 Cannabidiol [7] in Kombination mit Clobazam sowie seit 2021 Fenfluramin zur Verfügung [8]. Trotz patientenindividueller Kombinationstherapien sind die wenigsten Betroffenen anfallsfrei. Eine frühzeitige optimierte Therapie kann langfristig zu einer verbesserten Anfallskontrolle führen. Dass kognitive Leistungen oder Verhaltensauffälligkeiten dadurch verbessert werden, wäre wünschenswert, ist aber noch nicht wirklich geklärt $[9,10]$.

Ziel der Querschnittsstudie „Dravet syndrome caregiver survey" (DISCUSS) des europäischen Netzwerkes der "Dravet Syndrome European Federation" war es, Faktoren zu identifizieren und zu beschreiben, die einen Einfluss auf die Krankheitslast von Betroffenen mit DS und ihre Betreuer haben können [11]. In der vorliegenden Publikation werden die in Deutschland erhobenen Daten aus der Befragung von Eltern von am DS erkrankten Kindern vorgestellt.

\section{Methoden}

Die DISCUSS-Studie war eine anonym von der "Dravet Syndrome European Federation" durchgeführte europäische Querschnittsstudie auf Basis einer Befragung von Betreuern von Patienten mit DS im Zeitraum vom 23.06.2016 bis zum 04.08.2016. Die Methodik wurde ausführlich von Lagae et al. beschrieben [11]. Die Rekrutierung der Teilnehmer erfolgte in Deutschland durch die Patientenvertretung Dravet-Syndrom e. V. Es mussten 150 Fragen, die online zur Verfügung gestellt wurden, beantwortet werden.

Die Daten wurden als Gesamtzahlen, Häufigkeit der Antworten und zusammenfassende deskriptive Statistiken (Mittelwert und Standardabweichung [ \pm ; SD]) der gepoolten Stichprobe und der Altersgruppen für Kleinkinder ( $<2$ Jahre), Vorschulkinder ( 2 bis 5 Jahre), Grundschulkinder ( 6 bis 11 Jahre), Jugendliche (12 bis 17 Jahre) und Erwachsene ( $\geq 18$ Jahre) angegeben. Die gesundheitsbezogene Lebensqualität EQ-5D-5L wurde auf das deutsche Werte-Set umgerechnet. Höhere Indexwerte stehen für eine höhere Lebensqualität [11].

Hintergrund: Das Dravet-Syndrom (DS) ist ein seltenes, in der frühen Kindheit beginnendes, therapierefraktäres Epilepsiesyndrom, das mit einer hohen Morbidität und Mortalität verbunden ist.

Fragestellung: Ziele der Querschnittsstudie "Dravet syndrome caregiver survey" (DISCUSS) sind die Identifizierung und Beschreibung der Faktoren, die einen Einfluss auf die Krankheitslast von Patienten mit DS und ihre Betreuer haben können. Die Ergebnisse der deutschen Kohorte werden vorgestellt.

Material und Methoden: Die Datenerhebung erfolgte durch eine anonyme Befragung von Eltern. Die Ergebnisse wurden für die verschiedenen Altersgruppen statistisch ausgewertet.

Ergebnisse: Der Fragebogen wurde von 68 Eltern der DS-Patienten mit einem durchschnittlichen Alter von 10 Jahren (Median: 9, Spanne: 1-26) ausgefüllt. Nur 3 Patienten (4,4\%) waren in den letzten 3 Monaten anfallsfrei. Insgesamt hatten $97 \%$ der Patienten, die älter als 5 Jahre waren $(n=45)$, mindestens eine Komorbidität. Die zum Befragungszeitpunkt am häufigsten eingenommenen Antiepileptika waren Valproat, Kaliumbromid, Stiripentol, Clobazam und Topiramat. In der Vergangenheit wurden Natriumkanalblocker, Phenobarbital und Levetiracetam eingesetzt, aktuell fanden diese Antiepileptika nur selten Verwendung. Die Lebensqualität der Patienten war niedriger als die der Allgemeinbevölkerung. Die Erkrankung eines Familienmitglieds mit DS beeinflusst Eltern und Geschwister in hohem Maße.

Diskussion: Trotz individueller Kombinationstherapien sind die meisten Patienten mit DS nicht anfallsfrei. Insgesamt hat sich der Einsatz von beim DS wenig wirksamer Medikamente und der kontraindizierten Natriumkanalblocker zugunsten von wirksameren Medikamenten verschoben. Neue Therapie- und Versorgungskonzepte sind notwendig, um die Versorgung der Patienten mit DS zu verbessern und Eltern und Geschwister zu entlasten.

\section{Schlüsselwörter}

Enzephalopathie · Epilepsie · Epileptischer Anfall · Querschnittsstudie · Antikonvulsiva

\section{Ergebnisse}

\section{Demografie}

Insgesamt nahmen 68 deutsche Teilnehmer an der Umfrage teil. Das entspricht einem Anteil von 11,6\% an allen 584 europäischen Teilnehmern. $89,7 \%(n=61)$ der Antworten stammten von Müttern und $7,4 \%(n=5)$ von Vätern, $25,0 \%(n=17) \mathrm{der}$ Betreuenden waren alleinerziehend und $76,5 \%(n=52)$ Mitglied einer Selbsthilfegruppe oder Patientenvereinigung.

Das durchschnittliche Alter der Patienten lag bei 10 Jahren (Median: 9, Spanne: 1-26). 52,9\% $(n=36)$ der Patienten waren männlich. 8,8\% $(n=6)$ der Patienten waren Kleinkinder, 23,5\% ( $n=16)$ Vorschulkinder, 35,3\% $(n=24)$ Grundschulkinder, $19,1 \%(n=13)$ Jugendliche und 13,2\% ( $n=9)$ Erwachsene. 67,6\% $(n=46)$ der Betroffenen lebten mit mindestens einem Geschwister zusammen. Die Mehrzahl der Kinder und Jugendlichen $(81,1 \%, n=30)$ zwischen 6 und 17 Jahren besuchte eine Förderschule, $13,5 \%(n=5)$ besuchten ei- ne Regelschule und 5,4\% $(n=2)$ wurden zu Hause unterrichtet.

\section{Klinische Charakteristika}

\section{Zeit bis zur Diagnose}

Die Zeit bis zur Diagnosestellung hat sich innerhalb der letzten Jahre deutlich verkürzt. Während bei zum Zeitpunkt der Befragung Erwachsenen die Diagnose mindestens 4 Jahre erforderte, erhielten Vorschulkinder diese nach spätestens 2 Jahren und Kleinkinder nach spätestens einem Jahr. 61,8\% $(n=42)$ der Patienten erhielten vor der Diagnose DS eine bis 5 andere Diagnosen. Während $62,5 \%(n=10)$ der Vorschulkinder direkt die zutreffende Diagnose erhielten, war dies nur bei 22,2\% der Erwachsenen $(n=2)$ der Fall (• Abb. 1).

\section{Anfallstyp und -häufigkeit}

Insgesamt waren nur 3 Patienten (4,4\%) in den letzten 3 Monaten anfallsfrei. Mindestens einen tonisch-klonischen Anfall erlitten $86,8 \%(n=59)$ aller Patienten innerhalb der letzten 3 Monate, 63,2\% 


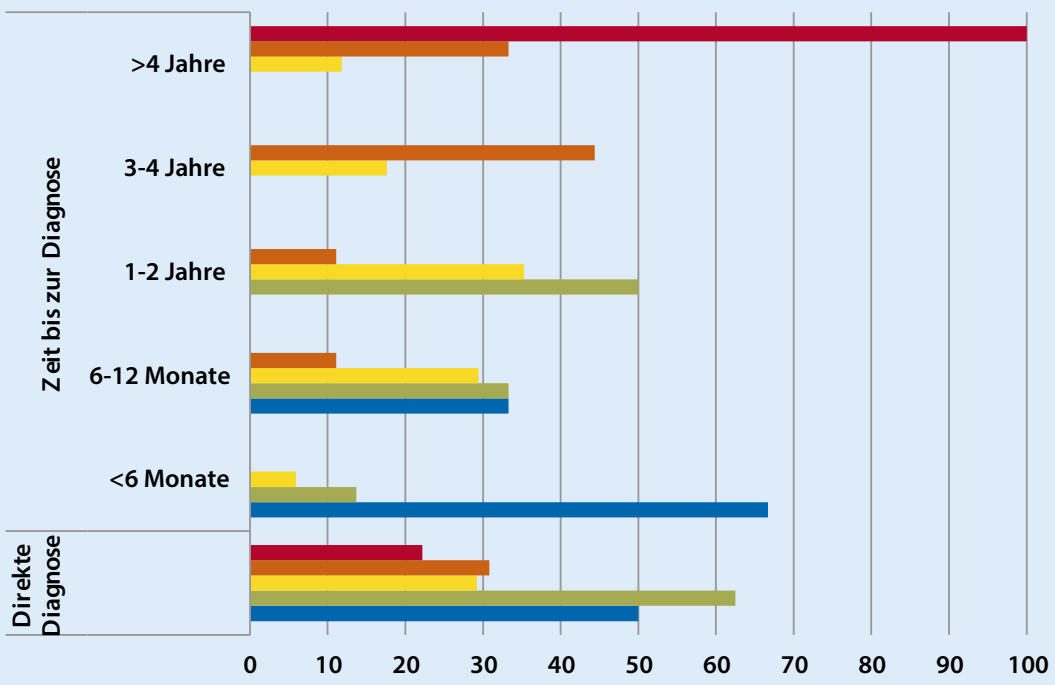

घrwachsene $\square$ Jugendliche Grundschulkinder $\backsim$ Vorschulkinder $\square$ Kleinkinder

Abb. $1 \Delta$ Zeit bis zur Diagnose eines Dravet-Syndroms in Abhängigkeit vom Lebensalter zum Zeitpunkt der Befragung

( $n=43)$ myoklonische Anfälle, 55,9\% ( $n=38)$ Absencen, 48,5\% ( $n=33)$ fokale Anfälle und $27,9 \%(n=19)$ atonische Anfälle/Sturzanfälle. Vor- und Grundschulkinder waren am häufigsten von mindestens einem Anfall innerhalb der letzten 3 Monate betroffen. Von den Vorschulkindern hatten 93,8\% $(n=15)$ mindestens einen tonisch-klonischen Anfall, 62,5\% $(n=10)$ Absencen oder fokale Anfälle und $43,8 \%(n=7)$ atonische bzw. Sturzanfälle. Unter den Grundschulkindern kam es bei $79,2 \%(n=19)$ zu myoklonischen Anfällen und bei $62,5 \%(n=15)$ zu Absencen. In dieser Altersklasse wurden mit 37,5\% $(n=9)$ häufig nichtklassifizierbare Anfälle berichtet. Bei allen Erwachsenen $(n=9)$ ist mindestens ein tonisch-klonischer Anfall innerhalb der letzten 3 Monate aufgetreten.

\section{Notfälle}

Mehr als die Hälfte aller Betroffenen $(55,9 \%, n=38)$ wurde in den letzten 12 Monaten mindestens einmal in der Notaufnahme behandelt, und 64,7\% $(n=44)$ riefen mindestens einmal den Rettungsdienst. Behandlungen in der Notaufnahme und Notrufe nahmen mit dem Alter zunächst $a b$ und stiegen bei Erwachsenen wieder an. So wurden alle Kleinkinder in den letzten 12 Monaten mindesten ein Mal in der Notaufnahme behandelt, $62,5 \%(n=10)$ der Vorschulkinder, 58,3\% $(n=14)$ der Grundschulkinder, $23,1 \%(n=3)$ der Jugendlichen und 55,6\% $(n=5)$ der Erwachsenen.

\section{Komorbiditäten}

Bei 97,0\% $(n=45)$ der Betroffenen, die älter als 5 Jahre waren, wurde mindestens eine Begleiterkrankung diagnostiziert. Im Schnitt wurden 4 von 6 abgefragten Begleiterkrankungen angegeben. Am häufigsten traten Lernschwierigkeiten $(91,3 \% ; n=42)$, Störungen der Motorik $(84,8 \% ; n=39)$ und Sprachstörungen $(67,4 \% ; n=31)$ auf. Bei $50 \%(n=23)$ wurde Autismus, bei $21,7 \%(n=10)$ ein Aufmerksamkeitsdefizit- und Hyperaktivitätssyndrom (ADHS) und bei 52,2\% $(n=24)$ wurden andere Verhaltensauffälligkeiten berichtet.

\section{Medikamentöse Therapie der Anfälle}

Die aktuell eingesetzten sowie jemals eingesetzten (Lebenszeitprävalenz) AED sind für alle Patienten und für die einzelnen Altersgruppen in - Tab. 1 dargestellt. Die Patienten erhielten zum Zeitpunkt der Umfrage im Durchschnitt 3 AED (SD 1,4; Spanne: 0-6, Median 3); in der Vergangenheit waren bereits durchschnittlich 3,2 AED (SD 3,0; Spanne: 0-11, Median 2) eingesetzt worden. Zu den am häufigsten aktuell eingenommenen AED gehörten Valproat $(63,2 \%, n=43)$, Kaliumbromid $(48,5 \%, n=33)$, Stiripentol $(44,1 \%, n=30)$, Clobazam $(39,7 \%, n=27)$ und Topiramat $(23,5 \%, n=16)$. In Bezug auf die Altersgruppen lässt sich kein einheitliches Muster erkennen; die Verordnung von Kaliumbromid ist nach Altersgruppen stratifiziert in - Tab. 2 abgebildet. 61,8\% der Patienten $(n=42)$ nahmen mindestens 3 AED ein (•Abb. 2), wie z.B. die Dreifachkombination aus Valproat, Stiripentol und Clobazam (20,6\%, $n=14$ ) (• Abb. 2).

In der Vergangenheit wurden andere Medikamente wie die Natriumkanalblocker Lamotrigin $(20,6 \%, n=14)$ und Oxcarbazepin $(14,7 \%, n=10)$, das Barbiturat Phenobarbital $(27,9 \%, n=19)$ sowie Levetiracetam $(44,1 \%, n=30)$ häufig eingesetzt. Zum Befragungszeitpunkt wurden sie sehr viel seltener oder gar nicht mehr eingenommen (•Tab. 1).

Neben den AED wurden bei den Patienten die Vagusnervstimulation (16,2\%, $n=11)$ oder eine ketogene Ernährungstherapie $(13,2 \%, n=9)$ eingesetzt. Die ketogene Diät war bereits bei $17,6 \%(n=12)$ früher eingesetzt worden.

\section{Therapie von Komorbiditäten}

Alle von motorischen Störungen oder Sprachstörung betroffenen Kleinkinder $(n=3$ bzw. $n=1)$ und $85,7 \% \quad(n=12)$ bzw. $66,7 \%(n=8)$ der Vorschulkinder erhielten eine Physio- oder Sprachtherapie, während andere Begleiterkrankungen in diesen beiden Altersklassen nicht oder nur selten therapiert wurden. Bei Patienten, die älter als 5 Jahre waren, wurden motorische Störungen oder Sprachstörungen bei $71,8 \%(n=28)$ bzw. $74,2 \%(n=23)$ therapiert, zudem wurde die Behandlung von Lernschwierigkeiten, Autismus, ADHS oder andere Verhaltensauffälligkeiten bei $16,7 \%,(n=7), 21,7 \%(n=5), 30,0 \%(n=3)$ bzw. 20,8\% ( $n=5)$ der Patienten berichtet.

\section{Lebensqualität}

Die Untersuchung der Lebensqualität ergab einen mittleren EQ-5D-5L-Index-Wert von 0,60 (Standardabweichung $\pm 0,26$ ) für Betroffene älter als 2 Jahre und liegt damit unterhalb des mittleren deutschen Indexwertes von 0,88 [12]. Zwischen den verschiedenen Altersgruppen gab es keine großen Unterschiede bezüglich der Le- 


\section{Originalien}

Tab. 1 Lebenszeitprävalenz aktuell und in der Vergangenheit eingenommener Antiepileptika (AED) beim Dravet-Syndrom

\begin{tabular}{|c|c|c|c|c|c|c|}
\hline & \multirow{2}{*}{$\begin{array}{l}\% \text { Antworten } \\
\text { in der gesam- } \\
\text { ten Kohorte }\end{array}$} & \multicolumn{5}{|c|}{$\%$-Antworten innerhalb der Altersgruppe } \\
\hline & & $\begin{array}{l}\text { Kleinkinder } \\
(n=6)\end{array}$ & $\begin{array}{l}\text { Vorschulkinder } \\
(n=16)\end{array}$ & $\begin{array}{l}\text { Grundschulkinder } \\
(n=24)\end{array}$ & $\begin{array}{l}\text { Jugendliche } \\
(n=13)\end{array}$ & $\begin{array}{l}\text { Erwachsene } \\
(n=9)\end{array}$ \\
\hline Patienten, die AED einnehmen & 100 & 100 & 100 & 100 & 100 & 100 \\
\hline \multicolumn{7}{|l|}{ Valproat } \\
\hline Aktuell & 63,2 & 83,3 & 75,0 & 58,3 & 53,8 & 55,6 \\
\hline IVE & 25,0 & 0 & 18,8 & 25 & 30,8 & 44,4 \\
\hline \multicolumn{7}{|l|}{ Clobazam } \\
\hline Aktuell & 39,7 & 50 & 43,8 & 41,7 & 30,8 & 33,3 \\
\hline IVE & 25,0 & 16,7 & 12,5 & 33,3 & 15,4 & 44,4 \\
\hline \multicolumn{7}{|l|}{ Stiripentol } \\
\hline Aktuell & 44,1 & 33,3 & 50,0 & 45,8 & 30,8 & 55,6 \\
\hline IVE & 20,6 & 16,7 & 6,3 & 29,2 & 23,1 & 22,2 \\
\hline \multicolumn{7}{|l|}{ Topiramat } \\
\hline Aktuell & 23,5 & 33,3 & 12,5 & 25,0 & 46,2 & 0 \\
\hline IVE & 25,0 & 0 & 12,5 & 25,0 & 23,1 & 66,7 \\
\hline \multicolumn{7}{|l|}{ Kaliumbromid } \\
\hline Aktuell & 48,5 & 16,7 & 62,5 & 54,2 & 38,5 & 44,4 \\
\hline IVE & 14,7 & 0 & 0 & 16,7 & 30,8 & 22,2 \\
\hline \multicolumn{7}{|l|}{ Cannabisprodukte } \\
\hline Aktuell & 5,9 & 0 & 0 & 16,7 & 0 & 0 \\
\hline IVE & 1,5 & 0 & 0 & 4,2 & 0 & 0 \\
\hline \multicolumn{7}{|l|}{ Carbamazepin } \\
\hline Aktuell & 1,5 & 0 & 0 & 4,2 & 0 & 0 \\
\hline IVE & 4,4 & 0 & 12,5 & 0 & 0 & 11,1 \\
\hline
\end{tabular}

\begin{tabular}{|l|l|l|l|l|l|l|}
\hline Aktuell & 0 & 0 & 0 & 0 & 0 & 0 \\
\hline IVE & 14,7 & 33,3 & 12,5 & 4,2 & 7,7 & 44,4 \\
\hline Phentoin
\end{tabular}

Phenytoin

\begin{tabular}{|c|c|c|c|c|c|c|}
\hline Aktuell & 0 & 0 & 0 & 0 & 0 & 0 \\
\hline IVE & 2,9 & 0 & 0 & 0 & 0 & 22,2 \\
\hline \multicolumn{7}{|c|}{ Lamotrigin } \\
\hline Aktuell & 1,5 & 0 & 0 & 0 & 0 & 11,1 \\
\hline IVE & 20,6 & 0 & 6,3 & 12,5 & 30,8 & 66,7 \\
\hline \multicolumn{7}{|c|}{ Vigabatrin } \\
\hline Aktuell & 0 & 0 & 0 & 0 & 0 & 0 \\
\hline IVE & 4,4 & 0 & 0 & 4,2 & 0 & 22,2 \\
\hline \multicolumn{7}{|c|}{ Phenobarbital } \\
\hline Aktuell & 0 & 0 & 0 & 0 & 0 & 0 \\
\hline IVE & 27,9 & 16,7 & 12,5 & 20,8 & 38,5 & 66,7 \\
\hline \multicolumn{7}{|c|}{ Rufinamid } \\
\hline Aktuell & 0 & 0 & 0 & 0 & 0 & 0 \\
\hline IVE & 1,5 & 0 & 0 & 4,2 & 0 & 0 \\
\hline \multicolumn{7}{|c|}{ Levetiracetam } \\
\hline Aktuell & 14,7 & 0 & 12,5 & 8,3 & 15,4 & 44,4 \\
\hline IVE & 44,1 & 66,7 & 56,3 & 37,5 & 38,5 & 33,3 \\
\hline \multicolumn{7}{|l|}{ Andere $^{a}$} \\
\hline Aktuell & 39,7 & 16,7 & 37,5 & 54,2 & 15,4 & 55,6 \\
\hline IVE & 45,6 & 0 & 31,3 & 54,2 & 46,2 & 77,8 \\
\hline
\end{tabular}

J. Jahre, Kleinkinder (<2 J.), Vorschulkinder (2-5 J.), Grundschulkinder (6-11 J.), Jugendliche (12-17 J.), Erwachsene ( $\geq 18 \mathrm{~J}$.$) , IVE in der Vergangenheit einge-$ nommen

aAndere Antiepileptika waren: Acetazolamid, Clonazepam, Diazepam, Ethosuximid, Felbamat, Gabapentin, Hydrocortison, Lacosamid, Mesuximid, Perampanel, Phenytoin, Sultiam, Zonisamid 
Tab. 2 Nach Altersgruppen stratifizierte Verordnung von Kaliumbromid in der europäischen und deutschen Kohorte

Kaliumbromid-Einnahme in \%

\begin{tabular}{|l|l|l|l|l|l|l|l|}
\hline \multicolumn{2}{|c|}{} & $\begin{array}{l}\text { \% Ge- } \\
\text { samt }\end{array}$ & K (n=6) & VS (n=16) & GS (n=24) & J (n=13) & E (n=9) \\
\hline $\begin{array}{l}\text { Euro- } \\
\text { päische } \\
\text { Kohorte }\end{array}$ & Aktuell & 9,6 & 8,8 & 11,3 & 10,9 & 9,3 & 5,0 \\
\cline { 2 - 8 } & IVE & 4,6 & 0,0 & 0,7 & 5,0 & 9,3 & 6,0 \\
\hline $\begin{array}{l}\text { Deutsche } \\
\text { Kohorte }\end{array}$ & Aktuell & 48,5 & 16,7 & 62,5 & 54,2 & 38,5 & 44,4 \\
\cline { 2 - 9 } & IVE & 14,7 & 0,0 & 0,0 & 16,7 & 30,8 & 22,2 \\
\hline
\end{tabular}

J. Jahre, K Kleinkinder (<2 J.), VS Vorschulkinder (2-5 J.), GS Grundschulkinder (6-11 J.), J Jugendliche (12-17 J.), E Erwachsene ( $\geq 18 \mathrm{~J}$.$) , IVE in der Vergangenheit eingenommen$

bensqualität mit einem Indexwert von 0,68 $( \pm 0,19)$ für Vorschulkinder, $0,59( \pm 0,33)$ für Grundschulkinder, 0,66 ( $\pm 0,19)$ für Jugendliche und 0,47 $( \pm 0,17)$ für Erwachsene.

\section{Ressourcenverbrauch}

Die jährlichen direkten Kosten pro Patient für die Behandlung anfallsbedingter Symptome (Notfallbehandlungen, Facharztbesuche, Medikamente) lagen bei insgesamt $7892 €$ und Kosten für die Therapie von Begleiterkrankungen bei $7271 €$ (Berechnungsgrundlagen: [13]). Selbst zu tragende Kosten wie etwa für die Pflege des Patienten lagen monatlich bei $75-400 €$ $(n=9$, Median $120 €)$. Zudem wurde weitere Ausgaben in Höhe von 10-6000€ ( $n=26$, Median $273 €$ ) für Hilfsmittel zur Behandlung motorischer Störungen (z.B. orthopädische Schuhe) angegeben. Indirekte Kosten aufgrund der Pflege des Patienten entstanden bei 40,0\% $(n=27)$ der Betreuer durch Lohnausfälle.

\section{Einfluss auf Familien}

\section{Betreuer}

Das tägliche Leben $(94,1 \%, n=64)$, familiäre Beziehungen $(76,5 \%, n=52)$ oder das Sozialleben $(70,6 \%, n=48)$ wurde/wurden durch die Betreuung eines Kindes, Jugendlichen oder Erwachsenen mit DS erschwert. Betreuer machten sich häufig Sorgen über die Zukunft des Kindes $(98,5 \%, n=67)$ oder die Verträglichkeit bzw. Wirksamkeit der medikamentösen Therapien (86,8\%, $n=59$ bzw. 69,1\%, $n=47$ ). Ihre eigene Berufsausübung sahen $86,0 \%(n=4)$ der Väter und $88,0 \%(n=54)$ der Mütter beeinflusst. Etwa ein Drittel der Betreuer kündigte den Arbeitsplatz, um sich um das eigene
Kind zu kümmern. Unterstützung erhielten Betreuer aus dem familiären Umfeld $(55,9 \%, n=38)$, von ihrem Partner $(42,6 \%$, $n=29)$, von Sozialdiensten $(11,8 \%, n=8)$ oder ambulanten privaten Pflegediensten $(23,5 \%, n=16)$.

\section{Geschwister}

Mehr als die Hälfte der Patienten mit DS hatte Geschwister, die im selben Haushalt lebten, aber oft von anderen Personen betreut wurden, während sich die Eltern um das Kind mit DS kümmerten. Insgesamt wurden 65,2\% $(n=30)$ der Geschwister in der Zeit von Familienangehörigen oder Freunden versorgt, während 10,9\% $(n=5)$ aufgrund ihres Alters keine Betreuung mehr benötigten. Auf Freizeitaktivitäten mussten $45,7 \%(n=21)$ der Geschwister in den letzten 4 Wochen verzichten.

\section{Diskussion}

Diese Querschnittsstudie liefert mit einer großen Stichprobe von 68 Patienten repräsentative Daten zum Einfluss des DS auf Patienten, Betreuer und Geschwister in Deutschland. Diese Ergebnisse der deutschen Kohorte werden im Folgenden mit den Ergebnissen der gesamteuropäischen Kohorte [11, 13] und denen einer anderen in 2017 prospektiv durchgeführten, multizentrischen Querschnittsstudie mit 93 befragten Eltern in Deutschland, die der vorliegenden Studie hinsichtlich demografischer und klinischer Basisdaten ähnelt, verglichen $[9,14,15]$.

Die Diagnose eines DS erfolgt klinisch und wird durch den Nachweis einer Mutation im SCN1A-Gen unterstützt; die Zeit bis zur korrekten Diagnose eines DS hat sich in den letzten Jahren in Deutschland signifikant verkürzt, wie in dieser Untersuchung und der 2017 Kohortenstudie gezeigt werden konnte [14]. Gründe könnten eine steigende Wahrnehmung des DS sowie ein guter Zugang zur neuropädiatrischen Versorgung und zur Gendiagnostik sein. Im Vergleich zur europäischen Kohorte erhielten deutsche Patienten früher die zutreffende Diagnose. Während alle Kinder unter 2 Jahren ihre Diagnose spätestens nach einem Jahr erhielten, war dies in der europäischen Kohorte bei 87,5\% der Fall. Aus der europäischen Kohorte erhielten 44,7\% der Vorschulkinder und $12,0 \%$ der erwachsenen Patienten direkt die Diagnose DS; in Deutschland waren es $62,5 \%$ bzw. 22,2\% [11]. Eine frühe, möglichst durch eine Gendiagnostik gestützte Diagnose des DS ermöglicht eine frühe, patientenindividuell optimierte Therapie sowie die Vermeidung kontraindizierter AED, sodass möglicherweise bessere Langzeitergebnisse erzielt werden können.

Die vorliegende Kohorte zeigte im Gegensatz zur europäischen Kohorte keine Korrelation zwischen dem jüngeren Alter der Patienten und einer hohen Anfallshäufigkeit bzw. der Häufigkeit von Notaufnahmen und Rettungseinsätzen. Auffällig waren insbesondere die höhere Anfallshäufigkeit und die häufigeren Notaufnahmen und Rettungseinsätze bei Erwachsenen, verglichen mit Jugendlichen [11]. Diese Ergebnisse können auf Schwierigkeiten in der Transition von der Neuropädiatrie in die Erwachsenenneurologie sowie beim Wechsel in die Wohn- oder Betreuungseinrichtung für Erwachsene hinweisen.

Charakteristisch für ein DS zeigten sich tonisch-klonische Anfälle bei allen Altersgruppen am häufigsten, sowie unterschiedliche andere Anfallssemiologien wie myoklonische Anfälle, Absencen und fokale Anfälle. Verglichen mit der europäischen Kohorte, in der sich 9,4\% innerhalb der letzten 3 Monate anfallsfrei zeigten, waren es in der deutschen Kohorte nur 4,4\% [11]. Letzteres war gut übereinstimmend zu der deutschen Querschnittsstudie aus 2017 mit 4,0\% (n=4) der Patienten, die eine Anfallsfreiheit von mehr als einem Jahr aufwiesen [9].

In der Lebenszeitprävalenz eingenommener AED beim DS zeigte sich ein breites Spektrum. Die durchschnittlich 3 (SD 1,4, Spanne 0-6) zum Erhebungszeitpunkt ein- 


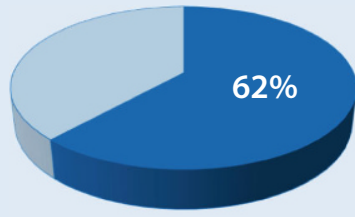

Drei AED oder mehr

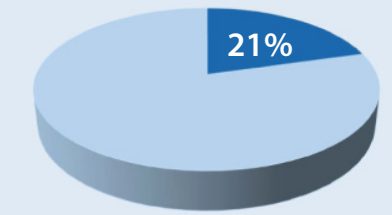

Kombinationstherapie Valproat, Stiripentol und Clobazam
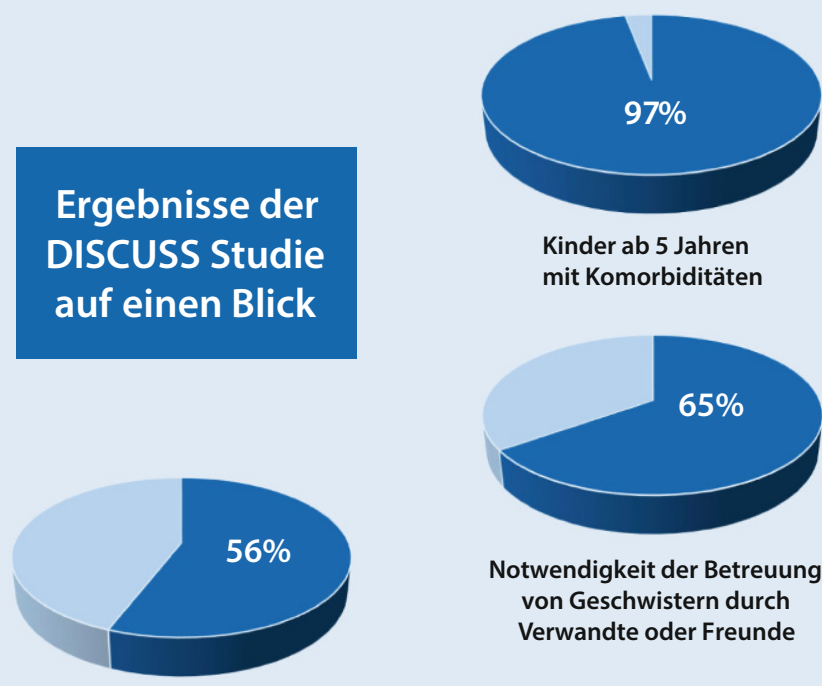

Behandlung in Notaufnahme in den letzten 12 Monaten

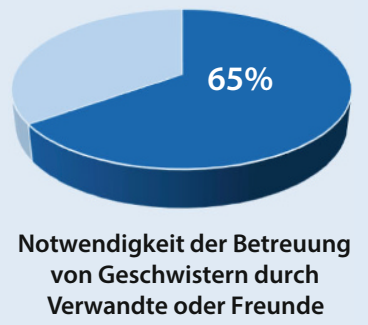

Abb. $2 \triangleleft$ Ergebnisse der deutschen Kohorte der DISCUSS-Studie (Dravet syndrome caregiver survey). $A E D$ ",antiepileptic drugs" genommen AED in der deutschen Kohorte waren mit den durchschnittlich 3,14 (SD 1,3, Spanne 0-12) der europäischen Kohorte vergleichbar, wobei die Spanne bei Letzterer deutlich breiter war. In der deutschen Querschnittsstudie aus 2017 lag der Durchschnitt mit 2,5 (SD 1,1, Spanne 0-6) etwas niedriger.

$\mathrm{Zu}$ den am häufigsten eingenommenen AED gehörten in der europäischen wie in der deutschen Kohorte Valproat (75,5\% bzw. 63,2\%), Stiripentol (47,1\% bzw. 44,1\%), Clobazam (52,7\% bzw. $39,7 \%$ ) und Topiramat (34,2 \% bzw. 23,5\%) [11], während Kaliumbromid (9,6\% bzw. $48,5 \%$ ) häufiger in der deutschen Kohorte eingenommen wurde. Die Ergebnisse decken sich mit den Ergebnissen der deutschen Querschnittsstudie aus 2017, in der diese 4 Medikamente $(66,0,35,0,41,0$ und $24,0 \%)$ und Kaliumbromid (44,0\%) ebenfalls die am häufigsten eingenommenen AED waren $[9,15]$. Der Einsatz von Kaliumbromid erfolgte in der deutschen Kohorte vorwiegend in der Altersgruppe der Vorschulkinder $(62,5 \%)$ und bei den Grundschulkindern (54,2\%, - Tab. 2). Dies lässt darauf schließen, dass Kaliumbromid erst nach Nichtansprechen auf andere AED im Kleinkindalter zum Einsatz kam. Bei den Vorschul- und Grundschulkindern liegt eine hohe Anfallslast vor, und dies lässt vermuten, dass Kaliumbromid als potentes AED deswegen so häufig eingesetzt wurde. Bei den Jugendlichen und Erwachsenen zeigt sich, dass bei ca. der Hälfte Kaliumbromid wieder abgesetzt wurde. Außerhalb Deutschlands war Kaliumbromid nur in Japan ein etabliertes AED [16] und ist aktuell außerhalb Deutschlands nur eingeschränkt verfügbar. In den internationalen Therapieempfehlungen von Wirrel et al. (2017) gab es keinen Konsens über die Wirksamkeit von Bromiden [17], es gibt jedoch eine gute Evidenzlage für den Einsatz des Medikamentes aus deutschen und japanischen Kohortenstudien [18-20].

In der Vergangenheit waren bei den Betroffenen mit DS bereits zahlreiche verschiedene Medikamente eingesetzt worden, die sich teilweise als nichtwirksam oder als kontraindiziert herausgestellt haben. Insgesamt hat sich der Einsatz beim DS wenig wirksamer Medikamente wie Levetiracetam und der kontraindizierten Natriumkanalblocker zugunsten von wirksameren Medikamenten verschoben. Da die Anzahl der durchschnittlich in der Vergangenheit eingenommenen AED im Vergleich zum Erhebungszeitraum nicht deutlich gesunken war, besteht nach wie vor schon bei jungen Patienten die Notwendigkeit einer Polytherapie, und dies ist ein Hinweis für den hohen therapeutischen Bedarf bei Patienten mit DS. Ansprechraten auf die verschiedenen Therapien sowie Gründe für einen Therapiewechsel oder -abbruch konnten in dieser Studie nicht beleuchtet werden.

Fast alle (91,0\%) Betroffenen, die älter als 5 Jahre waren, hatten mehrere Begleiterkrankungen. Am häufigsten traten motorische Störungen, Lernschwierigkeiten und Sprachstörungen auf. In der europäischen Kohorte entfiel der größte Anteil der Komorbiditäten auf Lernschwierigkeiten, die bei $98 \%$ der Patienten vorhanden waren. Darüber hinaus hatten $80,0 \%$ der Patienten Sprachstörungen, gefolgt von motorischen Störungen $(74,0 \%)$, sonstigen Verhaltensauffälligkeiten $(51,0 \%)$, Autismus (42,0\%) und ADHS $(24,0 \%)$. Von diesen Komorbiditäten wurden sowohl in der deutschen als auch der europäischen Kohorte v. a. motorische (71,8\% bzw. 68,0\%) und Sprachstörungen (74,2\% bzw. 70,0\%) therapiert $[11,13]$.

Aufgrund der Anfallshäufigkeit, der Vielzahl an Komorbiditäten und des therapeutischen Aufwands geht die Erkrankung mit einer deutlich reduzierten Lebensqualität einher. Alle Altersgruppen hatten niedrige Indizes. So lag z.B. der Index für Erwachsene mit 0,47 (SD 0,17) um 0,41 niedriger als der für die Allgemeinbevölkerung mit 0,88 [12, 21]. Auch auf europäischer Ebene zeigten sich keine großen Unterschiede zwischen den verschiedenen Altersgruppen. Der Mittelwert der deutschen Stichprobe $(0,60$, SD 0,27) lag über dem der europäischen Kohorte 
$(0,42$, SD 0,29$)$ [11]. Dies ist am ehesten darauf zurückzuführen, dass für die europäische Kohorte das britische Werte-Set verwendet wurde und die Ergebnisse der deutschen Kohorte auf das deutsche Werte-Set umgerechnet worden sind. In der deutschen Querschnittsstudie aus 2017 ergab die mit dem Kiddy-KINDL gemessene Lebensqualität signifikant niedrigere Werte für die Kinder mit DS im Vergleich zur allgemeinen Bevölkerung. Kinder zwischen 4 und 6 Jahren hatten eine um $20,6 \%$ (Werte 65,0 vs. 81,9 ) niedrigere Lebensqualität, bei Kindern zwischen 7 und 17 Jahren war sie um 29,4\% (Werte $54,4$ vs. 77,0$)$ niedriger [9].

Die für die europäische Kohorte aufgestellte Hypothese, dass die Anfallshäufigkeit mit dem Ausmaß kognitiver und Verhaltenseinschränkungen korreliert, wurde teilweise für die Gesamtkohorte bestätigt [11]. Die dafür notwendige Bildung von Strata war für die länderspezifischen Kohorten nicht präspezifiziert und bedarf zur Klärung der kausalen Zusammenhänge weiterer Studien [11].

Die finanziellen Aufwendungen für die Therapie eines Patienten mit DS sind als hoch einzustufen und dies konnte in mehreren Studien bestätigt werden [9, 22, 23]. Die von Lagae et al. (2019) berechneten durchschnittlichen jährlichen Kosten pro Patient lagen mit $7892 €$ für die Behandlung anfallsbedingter Symptome und mit $7271 €$ für die Therapie von Komorbiditäten über den durchschnittlichen Kosten der EU5 (Frankreich, Deutschland, Italien, Spanien, Großbritannien) mit 6783 und $6759 €[13]$. Für Familien und Betreuer sind teilweise zu entrichtende Eigenanteile und Kosten für zusätzliche Heilbehandlungen oder andere Therapien eine hohe finanzielle Belastung [23].

Die Erkrankung eines Familienmitglieds mit DS beeinflusst Eltern und Geschwister in hohem Maße. Dies bestätigt sich auch in der Studie von Strzelczyk et al., in der $38,2 \%$ der Betreuer im Vergleich zu 4,3\% der Normalbevölkerung über Symptome von Angst oder Depression (gemessen mit EQ-5D-3L) berichteten [9]. Der Anteil der Alleinerziehenden war mit $25 \%$ leicht höher, als für die Gesamtbevölkerung in Deutschland (19\%; www.destatis. de für 2017) angenommen wird. Geschwister können sich benachteiligt fühlen, da
Freizeitaktivitäten ausfielen oder ihre eigene Betreuung nicht durch die Eltern, sondern durch andere Familienmitglieder oder Freunde erfolgte.

Insgesamt lassen die zahlreichen Übereinstimmungen mit lediglich wenigen Unterschieden zwischen der europäischen Gesamtkohorte und der deutschen Teilkohorte auf länderübergreifend weitgehend vergleichbare Versorgungsstandards schließen.

Die Studie weist Limitationen auf. Insgesamt gehörten 76,5\% der Betreuer einer Selbsthilfegruppe oder Patientenvereinigung an. Dies könnte mit einem Bias, z.B. durch eine übermäßige Anzahl besonders informierter und überdurchschnittlich motivierter Betreuer verbunden sein. Die teilweise retrospektive Erfassung kann zu einem erhöhten Verzerrungspotenzial führen. Kleinkinder waren in der Studie unterrepräsentiert. Stratifikationsanalysen zur Abbildung von Zusammenhängen zwischen Krankheitsmerkmalen, Begleiterkrankungen, Lebensqualität und der Krankheitslast waren nur für die europäische Gesamtkohorte, nicht für die länderspezifischen Kohorten geplant.

\section{Fazit für die Praxis und Ausblick}

Eine frühzeitige Diagnose des DS, die anhand des klinischen Bildes gestellt und durch eine Gendiagnostik gestützt werden kann, ist wichtig, um ein optimales Patientenmanagement sicherzustellen.

Eine frühzeitige Therapie der Anfälle und Komorbiditäten ist von essenzieller Bedeutung zur Verbesserung der Langzeitergebnisse und hoffentlich auch der kognitiven Entwicklung. Noch ist nicht gesichert, ob eine Reduktion der Anfälle oder Status die geistige Entwicklung der Kinder positiv beeinflussen kann.

Eine engmaschige Unterstützung von Familien hilft bei der Bewältigung von krankheitsbedingten und sozioökonomischen Belastungen.

Es gibt derzeit keine kausale Therapie für das DS, mit der DS-bedingte Anfälle aller Patienten ausreichend kontrolliert werden können. Im Jahr 2019 wurde Cannabidiol in Kombination mit Clobazam für die Behandlung des DS zugelassen, seit Februar 2021 steht Fenfluramin zur Therapie des DS in Deutschland zur Verfügung.
Damit verbunden ist die Erwartung einer besseren Anfallskontrolle für einen relevanten Teil der Betroffenen.

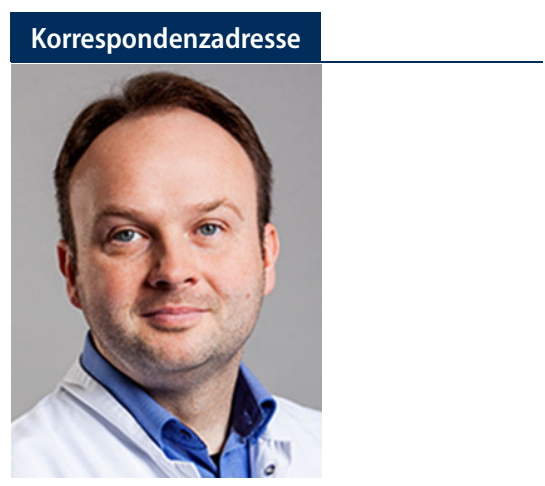

Prof. Dr. A. Strzelczyk

Epilepsiezentrum Frankfurt Rhein-Main, Zentrum der Neurologie und Neurochirurgie, Universitätsklinikum Frankfurt, GoetheUniversität Frankfurt

Schleusenweg 2-16, Haus 95, 60528 Frankfurt am Main, Deutschland

strzelczyk@med.uni-frankfurt.de

Funding. Open Access funding erfolgte mit freundlicher Unterstützung der Zogenix GmbH (München).

\section{Einhaltung ethischer Richtlinien}

Interessenkonflikt. A. Strzelczyk erhielt Beratungs-, Referentenhonorare und/oder Unterstützung für Forschungsvorhaben von Arvelle Therapeutics, Desitin Arzneimittel, Eisai, GW Pharma, LivaNova, Marinus Pharmaceuticals, Medtronic, UCB Pharma und Zogenix. L. Lagae erhielt Beratungs-, Referentenhonorare und/oder Unterstützung für Forschungsvorhaben von Zogenix, LivaNova, UCB, Shire, Eisai, Novartis, Takeda/ Ovid, NEL und Epihunter. L. Lagae besitzt ein Patent für ZX008 (Fenfluramin) zur Behandlung des DravetSyndroms und infantiler Epilepsien, das seiner Institution zugewiesen ist und an Zogenix lizenziert wurde. G. Kurlemann erhielt Beratungs- und Referentenhonorare von Desitin Arzneimittel, GW Pharma, Novartis, Takeda, UCB Pharma und Zogenix. T. Bast erhielt Honorare für Beratungs- und/oder Vortragstätigkeit sowie Aufwandsentschädigungen im Rahmen von Studien von Eisai, Desitin Arzneimittel, GW Pharma, Novartis, Nutricia, Shire, Takeda, UCB Pharma und Zogenix. T. Polster erhielt Beratungs-, Referentenhonorare und/oder Unterstützung für Forschungsvorhaben von Desitin Arzneimittel, Novartis, Shire, UCB Pharma und Zogenix. M. Pringsheim erhielt Beratungs- und Referentenhonorar von Zogenix. S. von Spiczak erhielt Beratungs- und Referentenhonorare und/oder Unterstützung für Forschungsvorhaben von Desitin Arzneimittel, Eisai und GW Pharma. P. Hipp erhielt ein Beratungshonorar von Almirall, MedDay, Sarepta und Zogenix. S. Schubert-Bast erhielt Beratungs-, Referentenhonorare und/oder Unterstützung für Forschungsvorhaben von Desitin Arzneimittel, Eisai, GW 
Pharma, UCB Pharma und Zogenix. S. Flege gibt an, dass kein Interessenkonflikt besteht.

Die Befragung erfolgte anonym durch das europäische Netzwerkder "Dravet Syndrome European Federation“ im Einklang mit der Deklaration von Helsinki von 1975 (in der aktuellen, überarbeiteten Fassung). Für die aufgeführten Studien gelten die jeweils dort angegebenen ethischen Richtlinien.

Open Access. Dieser Artikel wird unter der Creative Commons Namensnennung 4.0 International Lizenz veröffentlicht, welche die Nutzung, Vervielfältigung, Bearbeitung, Verbreitung und Wiedergabe in jeglichem Medium und Format erlaubt, sofern Sie den/die ursprünglichen Autor(en) und die Quelle ordnungsgemäß nennen, einen Link zur Creative Commons Lizenz beifügen und angeben, ob Änderungen vorgenommen wurden.

Die in diesem Artikel enthaltenen Bilder und sonstiges Drittmaterial unterliegen ebenfalls der genannten Creative Commons Lizenz, sofern sich aus der Abbildungslegende nichts anderes ergibt. Sofern das betreffende Material nicht unter der genannten Creative Commons Lizenz steht und die betreffende Handlung nicht nach gesetzlichen Vorschriften erlaubt ist, ist für die oben aufgeführten Weiterverwendungen des $\mathrm{Ma}$ terials die Einwilligung des jeweiligen Rechteinhabers einzuholen.

Weitere Details zur Lizenz entnehmen Sie bitte der Lizenzinformation auf http://creativecommons.org/ licenses/by/4.0/deed.de.

\section{Literatur}

1. Dravet C (2011) Dravet syndrome history. Dev Med Child Neurol 53(Suppl 2):1-6. https://doi.org/10. 1111/j.1469-8749.2011.03964.x

2. Gataullina S, Dulac O (2017) From genotype to phenotype in Dravet disease. Seizure 44:58-64. https://doi.org/10.1016/j.seizure.2016.10.014

3. Brunklaus A, Ellis R, Reavey E et al (2012) Prognostic, clinical and demographic features in SCN1A mutation-positive Dravet syndrome. Brain 135:2329-2336. https://doi.org/10.1093/brain/ aws151

4. Rosander C, Hallböök T (2015) Dravet syndrome in Sweden: a population-based study. Dev Med Child Neurol 57:628-633. https://doi.org/10.1111/ dmcn.12709

5. Bayat A, Hjalgrim H, Møller RS (2015) The incidence of SCN1A-related Dravet syndrome in Denmark is 1:22,000: a population-based study from 2004 to 2009. Epilepsia 56:e36-e39. https://doi.org/10. 1111/epi.12927

6. Wu YW, Sullivan J, McDaniel SS et al (2015) Incidence of Dravet syndrome in a US population. Pediatrics 136:e1310-e1315. https://doi.org/10. 1542/peds.2015-1807

7. Miller I, Scheffer IE, Gunning B et al (2020) DoseRanging Effect of Adjunctive Oral Cannabidiol vs Placebo on Convulsive Seizure Frequency in Dravet Syndrome. JAMA Neurology 77:613. https://doi. org/10.1001/jamaneurol.2020.0073

8. Nabbout R, Mistry A, Zuberi $S$ et al (2020) Fenfluramine for Treatment-Resistant Seizures in Patients With Dravet Syndrome Receiving Stiripentol-Inclusive Regimens. JAMA Neurology 77:300. https://doi.org/10.1001/jamaneurol.2019. 4113

\section{Clinical characteristics and quality of life with Dravet syndrome: results of the German cohort of the Dravet syndrome caregiver survey (DISCUSS)}

Background: Dravet syndrome (DS) is a rare treatment-resistant epilepsy syndrome with onset in infancy. It is associated with high morbidity and mortality.

Objective: The aims of the cross-sectional Dravet syndrome caregiver survey (DISCUSS) are to identify and describe those factors that may have an impact on the disease burden of patients with DS and their caregivers. The data of the German cohort are presented.

Material and methods: The data collection was based on an anonymous survey of parents, which was statistically analyzed for the different age groups.

Results: The questionnaire was completed by 68 caregivers of DS patients with a mean age of 10 years (median 9 years, range 1-26 years). In the previous 3 months only 3 patients (4.4\%) were seizure-free and $97 \%$ of the patients older than 5 years $(n=45)$ had at least 1 comorbidity. The antiepileptic drugs most commonly used at the time of the survey were valproate, potassium bromide, stiripentol, clobazam and topiramate. Sodium channel blockers, phenobarbital and levetiracetam have been used in past, but were given less frequently at the time of the survey. The patients' quality of life was lower than that of the general population. The disease of a family member with DS has a major impact on parents and siblings.

Conclusion: Despite individual combination therapies, most patients with DS are not seizure-free. Overall, the use of less effective drugs and of the contraindicated sodium channel blockers decreased and has shifted in favor of more effective drugs. New treatment and care concepts are needed to improve the care of patients with DS and to relieve the burden on parents and siblings.

\section{Keywords}

Encephalopathy · Epilepsy · Seizure · Cross-sectional study · Anticonvulsants

9. Strzelczyk A, Kalski M, Bast T et al (2019) Burdenof-illness and cost-driving factors in Dravet syndrome patients and carers: A prospective, multicenter study from Germany. Eur J Paediatr Neurol 23:392-403. https://doi.org/10.1016/j. ejpn.2019.02.014

10. Strzelczyk A, Schubert-Bast S (2020) Therapeutic advances in Dravet syndrome: a targeted literature review. Expert Rev Neurother 20:1065-1079. https://doi.org/10.1080/14737175.2020.1801423

11. Lagae L, Brambilla I, Mingorance A et al (2018) Quality of life and comorbidities associated with Dravet syndrome severity: a multinational cohort survey. Dev Med Child Neurol 60:63-72. https:// doi.org/10.1111/dmcn.13591

12. Grochtdreis T, Dams J, König HH, Konnopka A (2019) Health-related quality of life measured with the EQ-5D-5L: estimation of normative index values based on a representative German population sample and value set. Eur J Health Econ 20:933-944. https://doi.org/10.1007/s10198019-01054-1

13. Lagae L, Irwin J, Gibson E et al (2019) Caregiver impact and health service use in high and low severity Dravet syndrome: A multinational cohort study. Seizure 65:72-79. https://doi.org/10.1016/j. seizure.2018.12.018

14. Kalski M, Schubert-Bast S, Kieslich M et al (2019) Klinische Charakteristika, Ressourcenverbrauch, Lebensqualität und Versorgungssituation beim Dravet-Syndrom in Deutschland. Z Epileptol 32:326-338. https://doi.org/10.1007/s10309019-00287-7
15. Schubert-Bast S, Wolff M, Wiemer-Kruel A et al (2019) Seizure management and prescription patterns of anticonvulsants in Dravet syndrome: a multicenter cohort study from Germany and review of literature. Epilepsy Behav 98:88-95. https://doi.org/10.1016/j.yebeh.2019.06.021

16. Tanabe T, Awaya $Y$, Matsuishi T et al (2008) Management of and prophylaxis against status epilepticus in children with severe myoclonic epilepsy in infancy (SMEl; Dravet syndrome)-a nationwide questionnaire survey in Japan. Brain Dev 30:629-635. https://doi.org/10.1016/j. braindev.2008.03.002

17. Wirrell EC, Laux L, Donner E et al (2017) Optimizing the diagnosis and management of Dravet syndrome: recommendations from a north American consensus panel. Pediatr Neurol 68:18-34.e3. https://doi.org/10.1016/j. pediatrneurol.2017.01.025

18. Ernst J-P, Doose H, Baier WK (1988) Bromides were effective in intractable epilepsy with generalized tonic-clonic seizures and onset in early childhood. Brain Dev 10:385-388. https://doi.org/10.1016/ S0387-7604(88)80098-6

19. Oguni H, Hayashi K, Oguni Met al (1994) Treatment of severe myoclonic epilepsy in infants with bromide and its borderline variant. Epilepsia 35:1140-1145. https://doi.org/10.1111/j.15281157.1994.tb01780.x

20. Lotte J, Haberlandt E, Neubauer B et al (2012) Bromide in patients with SCN1A-mutations manifesting as Dravet syndrome. Neuropediatrics 43:17-21. https://doi.org/10.1055/s-00321307454 
21. Hinz A, Kohlmann T, Stöbel-Richter $Y$ et al (2014) The quality of life questionnaire EQ-5D$5 \mathrm{~L}$ : psychometric properties and normative values for the general German population. Qual Life Res 23:443-447. https://doi.org/10.1007/s11136013-0498-2

22. Strzelczyk A, Schubert-Bast S, Reese JP et al (2014) Evaluation of health-care utilization in patients with Dravet syndrome and on adjunctive treatment with stiripentol and clobazam. Epilepsy Behav 34:86-91. https://doi.org/10.1016/j.yebeh. 2014.03.014

23. Strzelczyk A, Schubert-Bast S, Bast T et al (2019) A multicenter, matched case-control analysis comparing burden-of-illness in Dravet syndrome to refractory epilepsy and seizure remission in patients and caregivers in Germany. Epilepsia 60:1697-1710. https://doi.org/10.1111/epi.16099

\section{Aktuelle Buchempfehlungen aus dem Springer-Verlag}

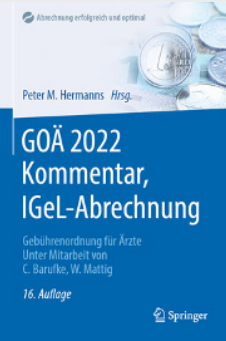

GOÄ 2022 Kommentar, IGeL-Abrechnung

\section{Gebührenordnung für Ärzte}

Hermanns, Peter M. (Hrsg.)

XXV, 896 Seiten

2022, 16. Auflage

Springer-Verlag

ISBN 978-3-662-64485-0

$79,99 €$

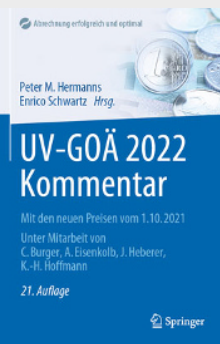

\section{UV-GOÄ 2022 Kommentar}

\section{Mit den neuen Preisen vom 1.10.2021}

Hermanns, Peter M., Schwartz, Enrico (Hrsg.)

XVII, 739 Seiten

2022, 21. Auflage

Springer-Verlag

ISBN 978-3-662-64487-4

$69,99 €$

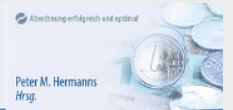

\section{EBM 2022 Kommentar}

Hermanns, Peter M. (Hrsg.)

EBM 2022

Kommentar

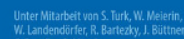

1. hantege XXVIII, 1004 Seiten

2022, 11. Auflage

Springer-Verlag

ISBN 978-3-662-64481-2

$79,99 €$

@springs

EBM 2022

Kommentar

Kinderheilkunde

rempitsmanaingroben

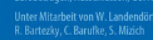

3. Ainfoge פิSpringer

\section{EBM 2022 Kommentar Kinderheilkunde}

Kompakt: Mit Punktangaben, Eurobeträgen, Ausschlüssen, GOÄ Hinweisen

Hermanns, Peter M. (Hrsg.)

XVII, 375 Seiten

2022, 3. Auflage

Springer-Verlag

ISBN 978-3-662-64483-6

$44,99 €$ 